\title{
Mapeando el (des)orden espacial: cartografía social en Cabure-í, Misiones
}

Francisco Fernández Romero

CONICET - Universidad de Buenos Aires. Facultad de Filosofía y Letras. Instituto de Geografía "Romualdo Ardissone". Buenos Aires, Argentina.

\section{Lucila Muñecas}

CONICET - Universidad de Buenos Aires. Facultad de Filosofía y Letras. Instituto de Geografía "Romualdo Ardissone". Buenos Aires, Argentina.

\section{Aymara Suyai Zanotti \\ CONICET - Universidad de Buenos Aires. Facultad de Filosofía y Letras. Instituto de Geografía "Romualdo Ardissone". Buenos Aires, Argentina. \\ Luis Piccinali \\ Universidad de Buenos Aires. Facultad de Filosofía y Letras. Departamento de Geografía - Instituto de Geografía "Romualdo Ardissone". Buenos Aires, Argentina.}

Recibido: 4 de noviembre de 2019. Aceptado: 9 de marzo de 2020.

\section{Resumen}

Nuestro objetivo central en este artículo es reflexionar sobre los aportes que puede realizar la cartografía social para identificar la espacialidad, o los aspectos específicamente espaciales, de los procesos sociales. Para ello, analizamos un mapeo realizado en Cabure-í, Misiones, junto con una cooperativa de pequeños productores. Este mapeo apuntaba a poner de relieve, para quienes integran la cooperativa, la dimensión espacial de la situación actual respecto a la tenencia de la tierra, lo cual contribuiría a re-enmarcar la precariedad en dicha tenencia no ya como un problema de familias particulares, sino como una problemática de escala colectiva. Pero, además, para nuestro grupo de investigación, esta actividad de cartografía social nos llevó a cuestionar nuestros propios supuestos en torno al orden espacial configurado por las y los integrantes de la cooperativa. Mientras que suponíamos que las divisiones catastrales oficiales sólo tendrían relevancia para la cotidianeidad de la población dado que legitiman órdenes de desalojo en su contra -casi ninguna familia es propietaria de la tierra-, hallamos que el mapa catastral, además, ha sido reapropiado por la población para la propia delimitación de sus chacras. 


\title{
Mapping spatial order and disorder: social cartography in Cabure-í, Misiones
}

\begin{abstract}
In this article, we aim to reflect on the contributions that social cartography or participatory mapping could make towards identifying the spatiality -or the specifically spatial aspects- of social processes. To this end, we analyze a mapping process we coorganized in Cabure-1́, Misiones, alongside a cooperative of small-scale farmers. This mapping aimed to highlight, for cooperative members, the spatial dimension of the current situation of land tenure in the area, which would enable to reframe precarious tenure situations as an issue on a collective scale, instead of as a problem pertaining to individual families. Furthermore, for our research team, this mapping caused us to question our own assumptions around the spatial order configured by the cooperative's members. While we assumed that the official cadastral land divisions would only be relevant to the population insofar as they legitimize eviction orders against these small farmers -almost none of which hold land titles-, we found that these farmers have also reappropriated the cadastral map to draw their own plot demarcations.
\end{abstract}

KEYWORDS: SOCIAL CARTOGRAPHY. CARTOGRAPHY. SPATIAL ORDER. CADASTRE. LAND TENURE.

PALAVRAS-CHAVE: CARTOGRAFIA SOCIAL. CARTOGRAFIA. ORDEM SPACIAL. CADASTRO. POSSE DA TERRA.

\section{Introducción}

La cartografía social es una metodología que implica la producción colectiva de mapas por fuera de los contextos institucionales convencionales. Viene siendo usada de manera creciente en ámbitos académicos y de extensión universitaria en Argentina, en paralelo a una aplicación cada vez mayor dentro del activismo y de la gestión estatal (Arzeno et al., 2017). En el proyecto de investigación que enmarca el presente artículo, nos hemos propuesto llevar adelante talleres de cartografía social con el fin de explorar las alternativas socio-espaciales planteadas por diferentes tipos de organizaciones sociales (campesinas, de la economía social, de personas con discapacidad, etc.) frente al orden espacial que se intenta imponer desde posiciones hegemónicas tales como el campo estatal. Esta propuesta de trabajo se vincula con en una serie de reflexiones que venimos realizando en torno a los vínculos entre investigadores y organizaciones sociales en el proceso de producción de conocimiento (Arzeno et al., 2017). Pero también nos interesa explorar la herramienta de la cartografía social como un modo de trabajar sobre los conflictos y alternativas desde una perspectiva específicamente espacial. Nos preguntamos, ¿qué posibilidades ofrece la cartografía social para resaltar los aspectos espaciales de las problemáticas que estudiamos? ¿En qué manera puede contribuir esta especificidad a nuestra investigación y a los fines de las organizaciones con las que trabajamos?

En este artículo, pretendemos sistematizar y analizar una experiencia en curso de mapeo con organizaciones, como forma de compartir preguntas y consideraciones que creemos pueden resultar útiles para otros sujetos interesados en esta herramienta. Específicamente, reflexionaremos sobre un mapeo realizado con la Cooperativa Cabure-í 
en el municipio de Andresito, norte de la provincia de Misiones. ${ }^{1}$ Dicha actividad fue planificada en conjunto con la cooperativa de manera tal que sirviera a los objetivos de la organización y de nuestras investigaciones. Sobre todo, fue pensada como un modo de sistematizar y espacializar datos vinculados a sus integrantes, particularmente con respecto a la situación de tenencia de la tierra, que es una problemática de suma relevancia en la zona debido al proceso actual de emisión de órdenes de desalojo a las y los habitantes que no poseen la propiedad legal de la tierra. Las y los dirigentes de la organización consideraban que esta sistematización y visualización de la información resultaría útil tanto para apoyar sus demandas frente al Estado como para fortalecer la organización interna.

Nuestro objetivo central es reflexionar sobre los aportes que puede realizar la cartografía social para identificar la espacialidad o los aspectos específicamente espaciales de los procesos sociales. Para ello, luego de presentar nuestro marco teórico-conceptual sobre espacio y cartografía, expondremos el contexto de la problemática en torno a la tenencia de la tierra en Cabure-í, para luego describir el mapeo allí realizado, lo que incluye dar cuenta de las decisiones metodológicas tomadas en distintos momentos: antes, durante y después de la instancia colectiva de mapeo. Luego, reflexionaremos en torno a cómo la cartografía social contribuyó a poner de relieve la dimensión espacial de la situación actual respecto a la tenencia de la tierra.

Como explicaremos, consideramos que el mapeo ha realizado aportes tanto para el grupo de investigación como para la organización social. Para nuestro grupo, la actividad incitó reflexiones en torno a las coincidencias y desencuentros entre la división de tierras propuesta "desde arriba" (expresado por ejemplo a través del catastro, en cuanto registro de la propiedad privada) y la división llevada a cabo "desde abajo" por la población rural organizada. Estas consideraciones contribuyen a las discusiones de nuestro proyecto marco sobre aquellas situaciones donde se producen tensiones entre las formas de "orden territorial" propuestas por diferentes actores. Por su parte, para las y los dirigentes de la cooperativa, el mapeo resultó útil para lograr el objetivo de que aquellas y aquellos participantes menos políticamente movilizados comenzaran a visualizar las problemáticas de tenencia de la tierra como parte de un proceso más amplio, con dimensión espacial, y no meramente como un problema de familias individuales.

\section{Marco teórico-conceptual}

En el presente artículo nos interesa explorar en profundidad los modos en que la cartografía social puede contribuir a indagar en los aspectos específicamente espaciales de los procesos sociales, en general, y en particular en las situaciones donde existen tensiones en torno al uso, ocupación o apropiación del espacio. Partimos de la base de que el mayor aporte específico de las herramientas cartográficas es que permiten representar relaciones espaciales (Lois, 2015). Es decir, los mapas sirven para ver -y por lo tanto comenzar a pensar en- este tipo de relaciones que no pueden ser inmediatamente percibidas por los sentidos: "el mapa [...] sirve para ver, para visualizar,

1 Dicha actividad se insertó en un proyecto de investigación con financiamiento UBACyT 2016 y 2018 y PICT 2015-2440 que se desarrolla en el Grupo de Estudios Geografías Emergentes: políticas, conflicto y alternativas socio-espaciales (GEm), con sede en el Instituto de Geografía de la UBA. 
para representarse mediante imágenes ópticas fenómenos de otro carácter, para imaginarse con rasgos visibles algo que no se tiene a la vista" (Lois, 2015:2). En particular, buscamos identificar los aportes de nuestro mapeo en Cabure-í hacia la comprensión de la espacialidad de las problemáticas de la zona tanto para el grupo de investigación como para la cooperativa. Para ello, hemos partido de cierta perspectiva teórica sobre el espacio, el orden espacial, la cartografía en general y la cartografía social en particular, que explicitaremos en la presente sección.

Siguiendo las discusiones desarrolladas en el campo de la geografía en el último medio siglo, y el "giro espacial" que se produjo en las ciencias sociales durante la década de 1990, partimos de la idea de que el espacio es una dimensión intrínseca de la vida social (Lefebvre, 2013 [1974]; Soja, 2016; Lopes de Souza, 2013). Es decir, la espacialidad -tanto como la temporalidad-participa de todo proceso social y, a la inversa, el espacio es producido socialmente en un proceso que no está exento de conflictos.

Partiendo de esa base, el proyecto marco dentro del cual se inserta la presente investigación busca analizar situaciones de tensión entre distintos actores en torno al modo de utilizar, ocupar o apropiarse del espacio. Estas situaciones son frecuentemente definidas desde ámbitos estatales y parte de la literatura académica como "problemas de ordenamiento territorial" en los cuales el Estado debe intervenir para alcanzar un orden espacial considerado óptimo, a partir de planes, normativas y otras propuestas que promueven ciertas formas de uso, ocupación y apropiación del espacio (Cabrales Barajas, 2006). Sin embargo, desde nuestra perspectiva, buscamos problematizar la cuestión del ordenamiento territorial, alejándonos de su definición habitual como instrumento de política pública que busca resolver situaciones de "desorden" espacial. Pensamos, en cambio, en el ordenamiento como una práctica intrínseca a la producción del espacio que es llevada a cabo por diferentes actores interesados en imponer un "orden" cuya definición siempre está en disputa (Arzeno, Muñecas y Zanotti, 2020; Haesbaert, 2014). En este sentido, las prácticas de ordenamiento no son potestad exclusiva del Estado ya que existen otras formas de ordenamiento promovidas desde la particularidad de los sujetos que las llevan a cabo y que, en algunos casos, tienden a cuestionar las formas hegemónicas de "orden".

De esta forma, se puede tender hacia la producción de un ordenamiento hegemónico como así también a un ordenamiento contrahegemónico. El orden espacial dominante tiende a reproducir las asimetrías estructurales de una sociedad y de un espacio capitalista; así, la idea de "desorden" es intrínseca a las prácticas de ordenamiento que materializan situaciones de exclusión, desigualdades, asimetrías, etc. Pero desde nuestra perspectiva, también pueden ser pensadas como otras formas de ordenamiento que cuestionan, resisten, "de-forman" o simplemente reacomodan esas prácticas dominantes de ordenamiento a sus necesidades concretas. En este sentido, creemos necesario introducir otro punto de vista que contemple estas formas de responder a ese ordenamiento dominante (Arzeno et al., 2020). Tal como detallaremos en el siguiente apartado, el proceso de cartografía social analizado en este artículo se desarrolló en el marco de una disputa por el "orden" espacial entre miembros de la Cooperativa Cabure-1́, por un lado, y por otro lado quienes detentan los títulos de propiedad de los lotes que dichos miembros ocupan. 
En el marco de nuestra investigación en general, y del caso de Cabure-í en particular, consideramos que los talleres de cartografía social constituyen una herramienta que permite explorar aquel "ordenamiento" que las organizaciones sociales (u otros actores) llevan adelante en el espacio o aquel que desearían para un futuro. La cartografía social -y otras prácticas cartográficas similares, tales como el mapeo participativo o el mapeo indígena- son metodologías que proponen plasmar colectivamente miradas sobre el espacio que difieren de aquellas que aparecen en las representaciones oficiales (Arzeno et al., 2017). Estos tipos de prácticas cartográficas han sido utilizados, entre múltiples otras cuestiones, para investigar y contribuir a procesos de lucha por el reconocimiento de tierras campesinas (GEPCyD, 2011) y/o de tierras indígenas (Salamanca, 2012). La cartografía social se enmarca en un universo más amplio de metodologías, como la investigación-acción participativa o la educación popular, que buscan transformar la relación entre investigadores e investigados al poner en valor los saberes y las capacidades de reflexión y de acción de éstos últimos (Fals Borda, 1979; Freire, 2008 [1970]). Estas prácticas cartográficas, generalmente, tienen el fin de poner en evidencia problemas o injusticias sociales y/o contribuir a generar algún cambio en esas situaciones (Diez Tetamanti, 2018). De esta manera, la cartografía social puede tener una función pedagógica, investigativa o política, o alguna combinación de las tres.

En algunas reflexiones sobre cartografía social se menciona que el trabajo políticopedagógico de esta metodología está vinculado con lo espacial o con lo territorial, aunque dichas ideas no lleguen a ampliarse. Por ejemplo, Habegger y Mancila (2006:6) enmarcan la cartografía social dentro de una "pedagogía del territorio" que "[abre] perspectivas para una mejor comprensión de la realidad territorial, de cómo vivimos el territorio que habitamos, y cómo construimos el futuro territorio que deseamos". Por su parte, Estrada (2010:12) sostiene que la cartografía social sirve para "vincular a la organización con la dimensión territorial, ponerla en debate con herramientas nuevas que puedan ser un insumo para una estrategia que se piense también territorial”.

Volviendo a las ideas planteadas más arriba, sobre ordenamientos contra/hegemónicos, podríamos afirmar que la cartografía social contribuye a revelar, por contraste, el "ordenamiento" que subyace implícitamente en las cartografías convencionales (las y los practicantes de estas metodologías frecuentemente retoman afirmaciones sobre la no-neutralidad de los mapas, tales como las de Harley, 2005). Simultáneamente, las cartografías sociales muestran la existencia de propuestas alternativas de "ordenamiento": revelan formas organizativas del espacio que emergen de lo cotidiano o desde la organización política. Por ejemplo, en nuestro caso, el mapeo evidencia el uso que las y los integrantes de la Cooperativa Cabure-í efectivamente le dan a la tierra, en contraste con la asignación de tierras prescripta por el catastro.

En este artículo, al indagar en el proceso de cartografía social desarrollado en Cabure-í, partimos de una comprensión procesual de la cartografía tal como la que proponen Kitchin y Dodge (2007). Desde esta perspectiva, el mapa no posee estabilidad ontológica, no es una imagen fija plasmada en un papel ni en una pantalla, sino que los mapas "son del momento, son traídos a la existencia a través de prácticas (encarnadas, sociales, técnicas), son siempre rehechos cada vez que se trabaja con ellos; [...] son transitorios y efímeros, siendo contingentes, relacionales y dependientes del contexto" (Kitchin y Dodge, 2007:335). Los mapas emergen en el marco de procesos -siempre contextualizados- denominados mapeos, que son "prácticas espaciales puestas en acto para 
resolver problemas relacionales (por ejemplo, cómo crear una mejor representación espacial, cómo entender una distribución espacial, cómo trasladarse de A a B, y así sucesivamente)" (Kitchin y Dodge, 2007:335).

Esta perspectiva nos resulta productiva en dos sentidos. En primer lugar, si todo mapeo se realiza para resolver algún tipo de problema espacial, surge la pregunta ¿̇qué problemas espaciales contribuyó a elucidar la experiencia cartográfica en Cabure-í? ¿Qué relaciones espaciales permite ver? Al organizar la actividad planteamos ciertos problemas espaciales a abordar, pero ¿qué otros problemas (y por lo tanto qué otros mapeos) surgieron, que no hubiésemos previsto? En segundo lugar, desde esta postura teórica, ninguna representación espacial es de manera estable un mapa, incluso si la imagen en sí permanece idéntica: emerge un mapa nuevo cada vez que se trabaja con dicha representación, es decir, cada vez que la misma forma parte de una nueva práctica de mapeo. Como veremos más adelante, esta consideración ha resultado productiva para reflexionar sobre los usos oficiales y alternativos que se le ha dado al mapa catastral de la zona.

\section{Situando la problemática de tierras en Cabure-í}

La experiencia de cartografía social fue realizada en el marco de uno de los casos de estudio contemplados en el proyecto de investigación más amplio; dicho caso gira en torno a una política de promoción sustentable de alimentos impulsada en los últimos diez años por el Parque Nacional Iguazú en Cabure-í, área vecina al Parque. Uno de los objetivos centrales de ese estudio de caso $^{2}$ tiene que ver con analizar las particularidades de estas iniciativas que entendemos como ordenamientos, en las cuales la dimensión ambiental juega un papel central. Partiendo del supuesto de que la conservación de la naturaleza es una actividad que involucra y gestiona intereses concretos, particulares, de ningún modo pensables como "comunes a todos" (ya que como cualquier política supone una asignación y redistribución de recursos, y con ello de poder, entre los actores que conforman el territorio), dicha investigación busca explorar de qué manera estas políticas, estos ordenamientos, contribuyen a la construcción (ya sea la reproducción o la eventual transformación) del orden territorial actualmente existente en el norte de la provincia.

El paraje Cabure-í está ubicado en el oeste del municipio de Andresito (departamento Gral. Belgrano), en el extremo norte de la provincia. Si bien el municipio como tal tiene una historia reciente (fue colonizado en la década de 1980 a través de un plan implementado desde la dictadura cívico-militar), la zona de Cabure-í fue de las primeras en ser pobladas, con anterioridad al plan de colonización. Su historia está marcada por los vaivenes de la industria maderera: en 1943 la empresa Queiroz instaló en la zona un aserradero y una laminadora, dando origen a una villa obrera; tres décadas después, la empresa cerraría esos establecimientos, trasladando parte del personal a una nueva planta en Eldorado y quedando otra parte desempleada (Scalerandi, 2011). Gran parte de los habitantes actuales de Cabure-í son ex trabajadores de la empresa (o sus hijos),

2 Se trata de la investigación desarrollada por Lucila Muñecas, "Políticas de conservación en el norte de Misiones (2008-2018): los valores del verde y la naturaleza que (se) ordena”, con beca interna doctoral del CONICET (2015-2020). 
que ante el despido resolvieron quedarse y producir en las tierras que habitaban. Fue recién a inicios de la presente década que, en un contexto de revalorización de las tierras por el avance de la actividad yerbatera y ganadera (y, en menor medida, turística), tuvieron lugar las primeras órdenes de desalojo de algunos de estos productores, evidenciándose un conflicto por la tierra hasta entonces latente.

Como mencionamos, Cabure-í se encuentra ubicada en el límite este del Parque Nacional Iguazú (PNI), dentro de lo que el mismo considera su zona de amortiguamiento. ${ }^{3}$ Esto hace de Cabure-í un área de intervención donde han venido desplegándose diversos proyectos y programas con el fin de volver a las actividades productivas más conciliables con los objetivos de conservación. La mayoría de los productores de la zona cultivan tabaco (actividad de impacto en términos ambientales y de salud para las y los productores); el objetivo principal de las intervenciones del PNI en la zona es promover la reconversión productiva hacia otro tipo de cultivos. La Cooperativa Cabure-í surge, en parte, como resultado de las políticas del PNI en la zona. Decimos que "en parte" porque, si bien es importante destacar el saldo organizativo como uno de los resultados de aquella política, el grado de organización y movilización política que hoy tiene la cooperativa dista mucho de los objetivos inicialmente concebidos desde la administración del Parque. De hecho, en gran medida debido a la problemática de tierras, ${ }^{4}$ ya no existe una articulación tan fuerte entre el PNI y la cooperativa. La injerencia del Parque en la zona se limita, hoy en día, a actividades de educación ambiental -mayormente en escuelas-, quedando a un lado el trabajo directo con productores.

En la actualidad, la cooperativa está integrada por más de 70 socios, con distinto grado de participación. Mientras que algunas personas están implicadas sólo en los proyectos productivos (elaboración de dulces y de alimentos derivados de mandioca, vivero de plantas nativas) o vinculados a lo productivo (construcción de galpones y otras infraestructuras), otras participan tanto de estos proyectos como de actividades más explícitamente político-organizativas: reuniones, asambleas, instancias de articulación a escala provincial y nacional, y movilizaciones en distintos puntos del país.

\section{Metodología}

Nuestra propuesta de realizar un trabajo de cartografía social llegó en un momento de pleno crecimiento para la cooperativa y la organización. En una de las entrevistas, realizadas en 2016, comentamos con las y los representantes de la organización la posibilidad de iniciar un proyecto de mapeo en conjunto. De nuestro lado, el interés tenía que ver

3 Desde hace ya varias décadas, el PNI reconoce al límite este como uno de "los más problemáticos (...), ya que casi en su totalidad está en contacto con terrenos privados con intensa actividad agropecuaria" (Parque Nacional Iguazú, 2017:315). Si bien no existe una normativa que restrinja por vía legal los usos y actividades en esta zona (como sí existe, por ejemplo, en la Reserva de Biósfera Yabotí, en el centro de la provincia), es intención del PNI generar allí, de hecho, una zona de amortiguamiento. En otro trabajo (Arzeno, Muñecas, Zanotti, 2020) se analizan los motivos que llevan al PNI a concentrarse en esta zona, existiendo otra área limítrofe (denominada Península) también con intensa actividad agropecuaria -principalmente, yerbatera-, pero con productores más grandes, capitalizados y con situación de tenencia regular.

4 Funcionarios del Parque entrevistados afirman que los lineamientos de intervención en la zona de Cabure-í varían notablemente según quién esté a cargo de la intendencia (máxima autoridad). Mientras que en la intendencia anterior existía un apoyo marcado al trabajo con los productores de la zona, la actual se inclina a definir dicha área como "excesivamente conflictiva", limitando el accionar del Parque en la misma. Algunos de los funcionarios entrevistados, de hecho, identifican a los productores de manera descalificativa en términos de "intrusos". 
con habilitar una forma más de conocer el trabajo que la cooperativa venía realizando en la zona: imaginábamos el mapeo como una herramienta que nos permitiría construir información sobre el vínculo de los y las productores/as con el territorio y los recursos, en particular, sobre la problemática de la tenencia de la tierra -que conocíamos hasta el momento por la referencia a casos puntuales- de manera conjunta y espacializada.

$\mathrm{Al}$ hacer nosotras/os la propuesta, las y los referentes respondieron positivamente, encontrando en la herramienta de cartografía social y en la experiencia en sí varias potencialidades. En primer lugar, serviría para sistematizar y espacializar información referida a sus integrantes, relativa tanto a aspectos generales (su número, actividad productiva, antigüedad en la zona) como, en particular, a la situación de tenencia de la tierra, problemática de suma relevancia en la zona. Según nos informaron las y los referentes de la organización, en años anteriores habían existido ofertas por parte del Municipio de realizar un censo en la zona, pero la iniciativa nunca llegó a concretarse. Nuestros interlocutores en esa ocasión manifestaban que el tener esa información recabada y sistematizada podría serles de utilidad tanto para conocerse mejor entre ellas y ellos mismos como para poder mostrar datos precisos y actualizados a la hora de solicitar financiamientos o para disputar espacio en situaciones de conflicto.

En segundo lugar, consideraban que el proceso de mapeo podría constituir en sí mismo, en tanto proceso, una instancia de fortalecimiento de la organización, en el sentido de motivar la participación de los y las socios/as no tan implicados, a la vez de llamar la atención y movilizarse en torno a una problemática sensible como es la cuestión de la tierra. Los y las referentes insistían en el hecho de que, dado que el conflicto ya estaba teniendo expresiones visibles (órdenes de desalojo y judicialización del conflicto), era de suma importancia alertar al conjunto de los socios sobre el problema, de cara a fortalecer a la organización en este aspecto y poder así, precisamente, organizar la resistencia.

\section{La preparación de los mapas base}

En los meses previos al taller, se preparó la cartografía a ser utilizada como "base" para el mapeo colectivo. Para esta cartografía de base, decidimos que nos resultarían relevantes dos tipos de atributos: por un lado, elementos de referencia tales como la red vial o los ríos - para facilitar que las y los participantes pudieran ubicarse- y por otro lado, las divisiones catastrales. En otras circunstancias, usar un mapa base con divisiones administrativas oficiales en una actividad de cartografía social podría haber sido irrelevante o incluso contraproducente, al imponer sobre las y los participantes una concepción del espacio que podría constreñir el mapeo. Sin embargo, en esta ocasión consideramos pertinentes las divisiones catastrales en tanto representación del ordenamiento territorial "oficial" que sustenta las órdenes de desalojo que han comenzado a recibir algunas/os habitantes y socias/os de la Cooperativa Cabure-í, las cuales forman parte de las preocupaciones que dieron origen al mapeo.

Para construir un mapa base con los dos tipos de atributos relevantes -el catastro y los elementos de referencia de la zona- decidimos trabajar con capas superpuestas de materiales: una imagen satelital impresa en papel sobre la cual colocaríamos un acetato transparente con el trazado de la red vial, los ríos y las divisiones catastrales (Figura 1). Al hacer coincidir el acetato con la imagen satelital de fondo, se facilitaría la identificación de elementos (ubicación de las casas, chacras, corrales, zonas de monte, 
división de lotes, etc.) por parte de las y los participantes. Sobre el acetato se imprimió información proveniente de organismos oficiales, consideradas estándares que aplican normas de calidad (ISO 19100) y que acuerdan el marco normativo de IDERA (Infraestructura de Datos Espaciales de la República Argentina). ${ }^{5}$

Otra decisión que se tuvo en cuenta fue el recorte del área a plasmar en el mapa base. Esto tuvo que ver con establecer un área rectangular en la que pudiera definirse el límite con coordenadas precisas para el área de estudio. Se consideró que la zona de mapeo abarcara Cabure-í y una porción del vecino PNI. El tamaño de hoja escogido para imprimir las imágenes satelitales y el acetato fue A1; este gran tamaño tenía el fin de facilitar el trabajo en grupos grandes. Además, la impresión del mapa fue a escala relativamente grande para poder identificar las pequeñas parcelas de las y los productores. Para abarcar toda la zona de interés con esta escala, la dividimos en varias hojas A1. Por otra parte, preparamos un mapa a menor escala (la misma superficie, representada en una imagen más pequeña) que cubría toda la zona, con el objetivo de utilizarla para una síntesis final como conclusión de la actividad.

El día anterior al desarrollo del mapeo, realizamos una reunión organizativa con la cooperativa para ultimar detalles. A partir de allí, elaboramos una serie de preguntas para realizar a cada familia; algunas de las respuestas serían mapeadas durante la actividad grupal, tales como la ubicación de sus parcelas o la situación con respecto a la tenencia de la tierra, mientras que otras preguntas se referían a dimensiones que no serían mapeadas pero que la organización deseaba sistematizar (por ejemplo, el tamaño de cada familia).

\section{El taller de cartografía social}

Uno de los requerimientos que propusieron desde la Cooperativa Cabure-í fue que el mapeo se realizara dentro del marco de una jornada de formación política, en la cual la actividad de cartografía social se combinaría con una serie de exposiciones y reflexiones sobre el tema de la propiedad y tenencia de la tierra en la zona y en América Latina en general, y sobre la conflictividad en torno a la disputa por la tierra en la que están involucrados algunos socios de la cooperativa frente a quienes detentan un título de propiedad sobre las tierras que éstos habitan. Las presentaciones y reflexiones le otorgaron un marco histórico y geográfico al mapeo en sí mismo, fundamental para la profundización en el análisis del conflicto; al tiempo que fue una oportunidad para reunir a la mayor cantidad posible de socios en un mismo espacio. Es preciso tener en cuenta que muchos de los y las integrantes de la cooperativa viven lejos de la sede y tienen que dejar sus tareas en la chacra para poder participar, con lo cual era importante para las y los referentes de la cooperativa con los que planificamos el taller poder aprovechar al máximo el encuentro.

La jornada se dividió en dos momentos. En el primero, se hizo una presentación de las y los participantes, en donde se mencionó la procedencia y su situación con respecto a la tenencia de la tierra. Había participantes de Cabure-í y de otro paraje denominado Sarandí, perteneciente a otro municipio, que en ese momento estaba en pleno conflicto

5 Se accedió a la información de catastro a través de la Infraestructura de Datos Espaciales de Misiones y a la información de rutas nacionales brindadas por el Instituto Geográfico Nacional (IGN). 
por amenazas de desalojo. A continuación, un invitado de la cooperativa realizó una extensa exposición sobre cómo fue el proceso de ocupación de la tierra en Latinoamérica a lo largo de los últimos siglos, para entender el surgimiento de la propiedad privada de la misma y de esta forma contextualizar históricamente el avance sobre la ocupación de lotes.

El segundo momento, correspondiente al mapeo en sí mismo, tuvo como objetivo cartografiar la situación con respecto a la tenencia de la tierra de los y las productores/as que estaban presentes y conocer las principales actividades que llevaban a cabo en cada chacra. ${ }^{6}$ La actividad requirió una división en grupos para el trabajo en profundidad. En cada uno de ellos, participantes de la cooperativa e integrantes del GEm trabajaron con imágenes y cartografía correspondientes a un recorte específico de la zona de Cabure-í (Figura 1). Con la ayuda de la imagen satelital y del acetato que contenía la delimitación del catastro provincial, cada familia localizó su chacra y trazó sus límites. Simultáneamente, cada familia completó un formulario con información sobre sus integrantes, actividad productiva y situación con respecto a la tenencia de la tierra. Este formulario funcionó como complemento de la información mapeada y como un censo de los integrantes de la cooperativa. A cada familia se le asignó un código de referencia que se colocó tanto en el mapa -identificando su chacra- como en el formulario completado. Además, se sombrearon de distintos colores las chacras, dependiendo la situación con respecto a la tenencia de la tierra; a saber: no poseen papeles, poseen título de propiedad o poseen permiso de ocupación.

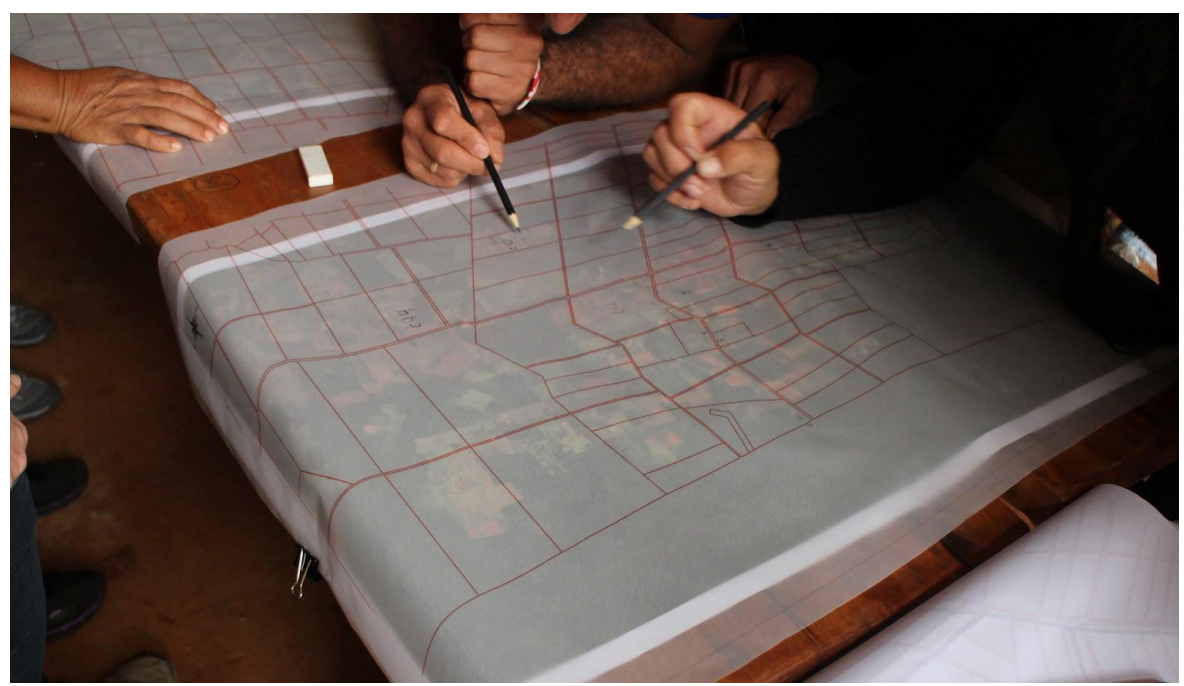

Figura 1. Instancia inicial del mapeo de las chacras. Fuente: fotografía propia.

Una vez finalizado el paso anterior, se procedió a hacer una puesta en común en donde la información mapeada por cada grupo se volcó en un único mapa, de menor

6 Como ya se mencionó, había compañeros/as de otro paraje; esto presentó una dificultad, porque en principio desde la organización del taller no estábamos enteradas/os de esto y por lo tanto no teníamos preparados mapas con el catastro de esta zona. De igual manera participaron de la actividad para que conocieran la herramienta y la pudieran replicar con sus compañeros/as y por otro porque también podían dar cuenta de un gran conocimiento sobre la zona y sus conflictos que era valioso para el mapeo. 
escala, que cubría toda la zona de interés (Figura 2). Este mapa había sido impreso solamente con las divisiones catastrales, rutas y ríos, dado que en esta instancia ya no era necesaria la imagen satelital para ubicarse. Además, se volvieron a delimitar las chacras de las y los participantes del mapeo, pero ya sin códigos que las asociaran a familias individuales. En cambio, solamente se colorearon sus parcelas en función de la situación con respecto a la tenencia de la tierra, destacando sobre todo la existencia de órdenes de desalojo. Esto permitió visualizar una situación generalizada de precariedad: casi ninguna familia poseía el título de las tierras y varias de ellas habían recibido órdenes de desalojo.

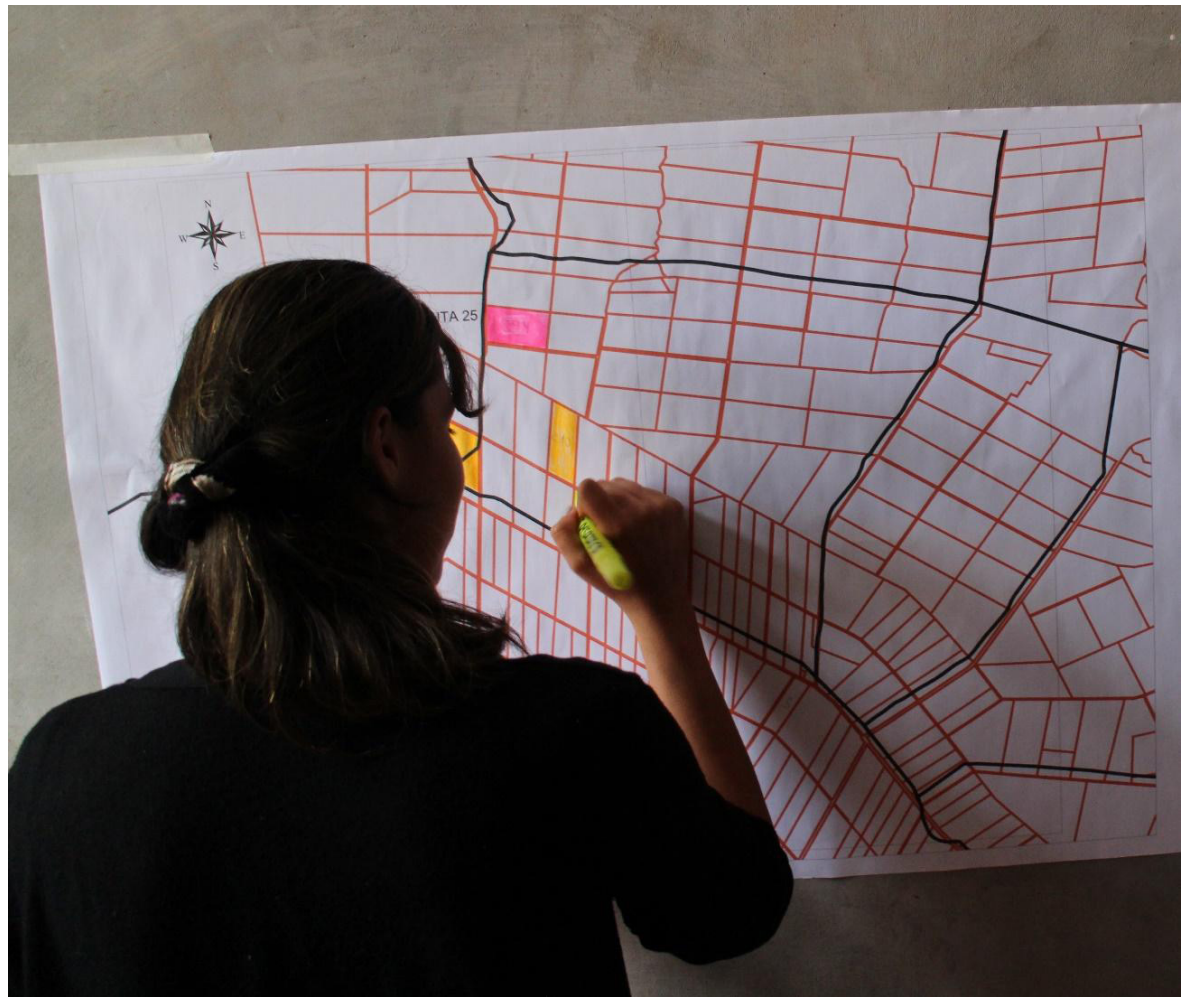

Figura 2. Mapa de la puesta en común. Fuente: fotografía propia.

Algunos aspectos relevantes surgieron del proceso de mapeo. El primero remite a la precisión con la que se delimitaron las chacras. Otro se refiere a que el catastro, de alguna manera, limita el formato de la chacra, porque ninguna excede los límites catastrales. Este punto nos resultó de relevancia para pensar cómo las divisiones catastrales parecen predefinir hasta cierto punto una espacialidad concreta, colocando límites (materiales y simbólicos) al proceso de ocupación, aunque sin determinar la propia ocupación y los acuerdos entre vecinos para delimitar sus chacras.

Otra cuestión de interés fue la puesta en común colectiva de la situación de tenencia de la tierra, ya que se pasó de un plano individual a otro grupal, en donde se identificaron situaciones similares que podrían permitir acciones conjuntas a futuro. Así, este pasaje a un mapa más general permitió trasladar la comprensión de las problemáticas de tenencia de la tierra a una escala mayor: ya no resultaba un problema privado, de familias individuales, sino una cuestión más amplia y colectiva. Justamente, es este 
aspecto del mapeo el que tiene la potencialidad de contribuir al objetivo de las y los dirigentes de la cooperativa de fomentar una mayor participación política de las y los socios menos implicadas/os.

\section{Después del taller}

En las reuniones preparatorias del taller, acordamos que luego de realizada la primera instancia de mapeo, comenzaríamos a cargar esa información -sumada a aquella que la organización fuese generando en mapeos posteriores- en un sistema web específico para ello. Hoy en día los recursos web para plasmar datos espaciales son innumerables (Open Street Map, Google Earth, Carto, etc.). La posibilidad que brindan estas herramientas es que permiten la georreferenciación de los datos relevados en un marco reconocido por los sistemas de información territorial y, por lo tanto, es fácil y ágil su incorporación una base de datos espaciales, cumpliendo con normas estándares (ISO 19100).

No obstante, utilizar estos sistemas implica una discusión o debate sobre la privacidad de los datos. Además, el uso de herramientas sistematizadas permite al participante establecer criterios de diseño sobre un mapa en la web, que no necesariamente son los más deseables o representativos. En él se establecen normas de estandarización de información geográfica que implican orientar el relevamiento o el conocimiento del territorio del mapeo colectivo a entidades gráficas espaciales preestablecidas. Eso supone una serie de discusiones con el fin de adaptar las representaciones que la comunidad local tiene sobre el espacio a las entidades geométricas y simbologías pensadas para el funcionamiento de un sistema de mapas web. A pesar de esto, a la Cooperativa Cabure-í le interesaba producir una salida informática con los datos recogidos. Finalmente, se acordó la generación y transferencia de una aplicación que no se guarda en línea, sino en el disco duro de la computadora, y que permite ver georreferenciada la información relevada colectivamente.

Actualmente, sin embargo, el proceso se encuentra en suspenso. La idea consensuada originalmente, que la cooperativa continuara por sus propios medios con el relevamiento y mapeo de las chacras de los habitantes que no hubieran participado en el taller, no fue llevada a cabo por el momento. Esto obedece a cuestiones que nos hablan, precisamente, de la dimensión político-organizativa que la herramienta de la cartografía social tiene para los dirigentes de la cooperativa. Como expusimos anteriormente, esta se encuentra en una etapa de crecimiento e incorporación de nuevas/os productoras/ es. Entendiendo que el relevamiento de información sobre chacras y productores (y en particular, sobre tenencia de la tierra) reviste un carácter sensible, desde la organización consideraron conveniente dar tiempo a que los nuevos/as socio/as se incorporaran más plenamente para, recién entonces, continuar con el proceso de mapeo.

\section{Aportes de la cartografía social para la reflexión espacial}

Como señalamos en la introducción, en este artículo buscamos reflexionar sobre las maneras en que la cartografía social puede contribuir a poner de relieve la dimensión espacial que consideramos es parte intrínseca de cualquier problemática social. Para nuestro grupo de investigación, esta experiencia de cartografía social sirvió para seguir fortaleciendo el vínculo con la cooperativa a partir del cual -antes, durante y después 
del mapeo- fuimos construyendo información que enriqueció nuestras investigaciones en la zona. Como hemos apuntado anteriormente sobre esta cuestión (Arzeno et al., 2017), aquí nos concentraremos en otro resultado de la actividad: nos sirvió como puntapié para comenzar a pensar sobre los modos en que el catastro se vincula con las prácticas espaciales de la población local.

Tal como ya mencionamos, en el trabajo que realiza nuestro equipo partimos de la idea de que existen ordenamientos territoriales "desde arriba" y "desde abajo" que disputan el territorio. El ordenamiento "desde arriba" se expresa y se posibilita, en parte, a través de la producción de divisiones geográficas oficiales que intentan asir el espacio para conocerlo, administrarlo, gestionarlo, etc. (Anderson, 2007 [1983]; Lois, 2014). Así, desde instituciones gubernamentales se producen diversas imágenes sobre el espacio, incluyendo los mapas oficiales que se ponen a disposición del público, donde este aparece fraccionado según los límites políticos oficiales. Pero también se producen otros mapas que se usan para operar dentro de instituciones estatales, en los cuales el espacio se divide siguiendo criterios tales como las unidades geoestadísticas (localidades/fracciones/radios censales) o las divisiones catastrales.

En los mapas base que preparamos para la actividad, incluimos las demarcaciones catastrales partiendo de la suposición de que ellas resultarían principalmente relevantes para comprender el ordenamiento territorial "desde arriba". Al fin y al cabo, los derechos legales de propiedad de la tierra que están plasmadas en el catastro son lo que se intenta imponer a través de acciones judiciales tales como órdenes de desalojo. Nuestra hipótesis era que dicha división legal de la tierra contrastaría con la delimitación de las parcelas realizada por las familias que efectivamente habitan en la zona, delimitación que suponíamos seguiría otros criterios de ordenamiento del espacio, sin tener en cuenta las delimitaciones catastrales. Es decir, suponíamos que los límites catastrales no serían relevantes para los y las habitantes en su cotidiano, sino que únicamente aparecerían en sus vidas al chocar con las fuerzas estatales y del mercado en la disputa por la tenencia de la tierra.

Múltiples autores han afirmado que la lógica espacial del capitalismo en general (Lefebvre, 2009 [1970]), y los registros catastrales en particular (Craib, 2013), operan en base a una concepción del espacio geométrica, homogeneizante, que hace abstracción de lo que realmente sucede en el terreno. Se trata de espacios donde "todo es equivalente, canjeable, intercambiable; pues es un espacio que es comprado y vendido, y el intercambio sólo puede ocurrir entre unidades que sean equivalentes, permutables" (Lefebvre, 2009 [1970]:233). Según Craib (2013), quien retoma la oposición que describe Bachelard entre el espacio del geómetra y el espacio del habitante, la creación de los registros catastrales sigue una lógica espacial que contrasta con la lógica y los usos propios de la población local; podríamos decir que no se condice con el espacio vivido, con el "orden" alternativo de los habitantes. Refiriéndose al proceso de agrimensura y reparto de tierras en el estado de Veracruz durante el siglo XIX, Craib señala que la división de tierras mandada por leyes federales y estatales negaba los derechos a la tierra previamente establecidos (los cuales frecuentemente variaban año a año y eran fuertemente contextualizados, según el tipo de terreno o de cultivo), reemplazándolos por una "lógica que ordenaba la tierra como un mosaico de lotes permanentes -científicamente medidos y limpiamente cartografiados-" (Craib, 2013:149). 
Existen claras diferencias entre ejemplos como el recién descripto y la situación de Cabure-í, ya que aquí la división catastral de tierras sucedió antes de la llegada de las y los habitantes actuales (aunque seguramente alteró las relaciones socio-espaciales previamente existentes). Pero suponíamos que podría existir una desconexión similar entre los límites catastrales y las prácticas cotidianas. Sin embargo, hallamos que las y los participantes del mapeo conocían la ubicación de las divisiones catastrales, a pesar de que no estén marcadas en el terreno de manera visible. De hecho, cada una de las parcelas ocupadas por las familias se alinea dentro de alguno de los lotes oficialmente delimitados; sus parcelas son más pequeñas que los lotes, pero ninguna cruza los límites de los lotes catastrales. Existe un desfasaje en tanto el catastro reconoce derechos de propiedad a individuos que no se vinculan cotidianamente con la tierra, pero las divisiones catastrales poseen relevancia en la manera en que las y los habitantes ocupan el espacio.

Podríamos pensar en esta situación como un ejemplo de ordenamiento territorial "desde abajo", entendido como aquellas prácticas de los habitantes que disponen elementos en el espacio de una manera que cobra sentido y coherencia en tanto reproduce un modo de vida subalterno. Lo denominamos "desde abajo" porque viene a cuestionar aquel ordenamiento territorial estatal que intenta ordenar aquello que se entiende como "desordenado". Arzeno (2019), siguiendo a Haesbaert, Lopes de Souza y Moreira, entiende que esas prácticas vistas como "desordenadas" cuestionan el ordenamiento territorial instituido como forma de vida hegemónica. Asimismo, comprende a estas prácticas subalternas de resistencia en dos sentidos: (i) organizadas y políticamente explícitas y (ii) prácticas cotidianas y aparentemente silenciosas, pero que cuestionan el ordenamiento territorial dominante. La autora retoma la definición de contraespacio de Moreira para referirse al ordenamiento territorial "desde abajo", entendiendo el contraespacio como "el modo espacial por medio del cual excluidos y dominados ponen en cuestión el orden espacial instituido como forma de organización de la sociedad, rechazando (o copiando) el modo de vida que ella impone." (Moreira, 2011, en Arzeno, 2019:13).

Teniendo en cuenta esta noción de contraespacio, nos han quedado abiertas varias preguntas: ¿Por qué las y los habitantes de Cabure-í decidieron tomar en cuenta las divisiones catastrales al trazar sus parcelas? ¿De qué manera lograron ubicar en el terreno las líneas divisorias entre lotes, dada la invisibilidad de las delimitaciones catastrales en el terreno? ¿Fue parte de una estrategia en la cual eventualmente planeaban perseguir la titularidad legal de las tierras, es decir, en la cual buscarían cuestionar la asignación de las relaciones de propiedad afirmadas por el catastro? ¿O fue parte de una estrategia alternativa?

Por otra parte, esta situación nos llevó a repensar las maneras en que las divisiones oficiales del espacio, tales como el catastro, producen efectos. Varios autores del campo de las cartografías críticas, tales como Pickles (2004) y Wood y Fels (2008), han afirmado que los mapas no son reflejos descriptivos de la realidad, sino que son instrumentos prescriptivos que producen realidad. Sin embargo, más recientemente, autores tales como Kitchin, Gleeson y Dodge (2012) han cuestionado esta afirmación ya que implica mantener el foco sobre el mapa en sí, mientras que ellos cuestionan la estabilidad ontológica del mapa como "cosa" que produce efectos por sí mismo. Sostienen que se debe enfocar más bien en las prácticas de mapeo en las cuales se insertan las imágenes del espacio, en vez de tomar a las imágenes cartográficas mismas como variable explicativa 
de los procesos espaciales (Kitchin y Dodge, 2007). Partiendo de esta postura, Fogelman y Bassett (2017) han investigado sobre el vínculo entre la actualización de los mapas catastrales en Lesotho y los procesos de mercantilización y desposesión de tierras. Afirman que los mapas en sí mismos no produjeron esos procesos, sino que los mapas han estado inscritos en una serie de prácticas sociales más amplias que contribuyeron a -pero no determinaron- que las tierras de ciertas zonas de Lesotho se volvieran más legibles y deseables para inversores.

Desde esta perspectiva, podemos considerar los modos en que el catastro de Cabure-í contribuye a producir realidad, pero no de manera determinante ni lineal. En efecto, si bien el mapa catastral produce cierta distribución legal de las tierras que permite el inicio de demandas por parte de los y las propietarios/as, la mera existencia del catastro no ha impedido la ocupación de las tierras por otros/as habitantes. Además, el catastro ha tenido efectos imprevistos y contraintuitivos, tales como la apropiación creativa que las y los habitantes han hecho del mismo: ellas y ellos han adoptado los límites catastrales para demarcar sus propias tierras, al mismo tiempo que rechazan la asignación de propiedad que el catastro implica.

Este es un ejemplo más de que un mapa -incluso un mapa oficial- no produce automáticamente una realidad por sí mismo, sino que es necesario pensar en mapeos (en términos de Kitchin y Dodge, 2007) en los cuales una misma imagen del espacio -en este caso, el mapa catastral- puede contribuir a producir diferentes realidades de acuerdo con las prácticas espaciales en las cuales se inscriba. Pensar en prácticas o procesos de mapeo también permite entender que, cuando se trata de mapas, pueden existir múltiples apropiaciones e interpretaciones ya que la agencia no yace únicamente en quien trazó el mapa (el cual, además, no es un actor individual ni homogéneo: generalmente están implicados uno o varios equipos con intereses posiblemente contradictorios, Fogelman y Bassett, 2017).

\section{Reflexiones finales}

Si retomamos la afirmación de Lois (2015) de que los mapas permiten ver relaciones espaciales que no son directamente percibibles por los sentidos, podemos preguntarnos ¿qué ha permitido ver el proceso de mapeo en Cabure-í? Si, como afirman Kitchin y Dodge (2007), los mapeos siempre apuntan a resolver un problema espacial, ¿qué problemas espaciales se han encarado en este mapeo?

Por un lado, como hemos mencionado, las y los integrantes de la Cooperativa Cabure-í con quienes organizamos el mapeo estaban interesadas/os en el mismo porque esperaban que una mayor cantidad de socias/os tomara conocimiento y consciencia de la dimensión de las problemáticas de tenencia de la tierra. En ese sentido, nuestra intención consistía en que el mapeo les sirviera para que incentivaran a las y los socias/ os con menor grado de participación a que comenzaran a visualizar las problemáticas de tenencia de la tierra como una problemática colectiva, con dimensión espacial. En efecto, consideramos que la espacialización de estas situaciones sirvió para dejar de pensar en las amenazas de desalojo como algo que solamente afecta a familias individuales, para pasar a concebirlo como un proceso que ocurre a otra escala; esto, a su vez, podría servir para incentivar la organización y la resistencia a una escala colectiva. 
Además, como hemos descripto, el proceso de cartografiado fue precedido por una charla expositiva sobre la historia de la propiedad privada de la tierra en general, lo cual complementó la potencialidad del mapeo para pensar en las problemáticas de tierras a escalas más amplias.

Por nuestra parte, uno de los principales aportes que pensábamos obtener de la investigación era el fortalecimiento del vínculo con la Cooperativa Cabure-í. En efecto, la cartografía social posee fuerte potencialidad para construir vínculos (dentro de y entre la organización y nuestro grupo de investigación). Al fin y al cabo, es desde ese vínculo que se construye la información sobre el espacio, sobre las problemáticas, sobre la geografía. Resulta interesante pasar de pensar en el mapa como herramienta de producción de información, a pensar los mapeos como condiciones de posibilidad para la producción de información.

Pero la experiencia también nos llevó, de manera inesperada, a las reflexiones ya expuestas en torno a los contrastes y coincidencias entre el orden espacial propuesto "desde arriba" y "desde abajo". En este sentido, el buen orden o el orden deseable es el orden socio-espacial dominante, que se vincula con sentidos subjetivos e intersubjetivos sobre lo que es deseado (Lopes de Souza, 2013). El catastro es un ejemplo de herramienta que visibiliza un "buen orden" o un "orden deseado" del espacio, respondiendo a las formas que adopta el mercado de tierras formal y legal. Ese orden es el que se busca imponer en Cabure-í a través de órdenes de desalojo a aquellas/os habitantes que no poseen los títulos de las tierras. Nuestro interés se centró en poner en cuestión aquellas prácticas hegemónicas al tiempo que analizamos prácticas de resistencia que se vinculan con algún sentido alternativo de orden espacial. De esta forma, consideramos relevante poner a dialogar, en este caso a través de la cartografía social, el orden hegemónico (el del catastro) con el orden que se promueve desde la organización con respecto a las formas de habitar ese espacio, haciendo foco en ese espacio vivido. Desde el mapeo no sólo se muestran otras espacialidades, sino que se ponen de relieve tensiones, conflictos y transformaciones del espacio a partir de procesos sociales.

Así, el mapeo nos permitió ver relaciones (en este caso, continuidades entre el catastro y la ocupación real de las tierras) que no habíamos percibido previamente, a pesar de trabajos de campo previos en la zona. Nos incitó a profundizar nuestras discusiones en torno al binomio orden/desorden territorial, provocando reflexiones en torno al rol de las cartografías oficiales (en este caso, el mapa catastral) como herramientas que canalizan un "buen orden" o un "orden deseado" del espacio. Si bien estas cartografías responden a las formas que adopta el mercado de tierras formal y legal, y vuelven posible la imposición del orden espacial hegemónico (por ejemplo, al justificar las órdenes de desalojo hacia aquellas/os habitantes que no poseen los títulos de las tierras), estas mismas cartografías pueden ser usadas creativamente como parte de órdenes espaciales alternativos donde las líneas en la tierra son las mismas, pero los usos y apropiaciones son diferentes a los prescriptos. 


\section{Q Bibliografía}

" Anderson, B. (2007 [1983]). Comunidades imaginadas. Reflexiones sobre el origen y la difusión del nacionalismo. México DF: Fondo de Cultura Económica.

» Arzeno, M. (2019). Orden-desorden y ordenamiento territorial como tecnología de gobierno. Estudios Socioterritoriales. Revista de Geografía, 25, 1-16.

》 Arzeno, M, Fernández Romero, F., Jurado, E., Muñecas, L. y Zanotti, A. (2017). Políticas, prácticas y representaciones en relación con el ordenamiento del territorio, la agricultura familiar y la construcción de alternativas socio-espaciales. Casos en Argentina. En N. Finelli y M. Cardoso (Comp.), Temas de investigación y debate en la Ciencia Geográfica (pp. 389-407). Santa Fe: Universidad Nacional del Litoral. E-book.

》 Arzeno, M., Muñecas, L. y Zanotti, A. (2020). Ordenamiento territorial en cuestión: orden y contraespacio en el norte de Misiones (Argentina). Cuadernos de Geografía, Revista Colombiana de Geografía, 29(1). En prensa.

》Cabrales Barajas, L.F. (2006). Geografía y ordenamiento territorial. En D. Hiernaux y A. Lindón (Comps.), Tratado de Geografía Humana (pp. 601-627). México DF: Anthropos.

» Craib, R.B. (2013). México cartográfico. Una historia de límites fijos y paisajes fugitivos. México DF: Universidad Nacional Autónoma de México.

»Diez Tetamanti, J.M. (2018). Cartografía social: teoría y método. Estrategias para una eficaz transformación comunitaria. Buenos Aires: Biblos.

» Estrada, M. de (2010). "O te mapeas o te mapean": el papel del mapa en la geografía. Mimeo.

» Fals Borda, O. (1979). El problema de cómo investigar la realidad para transformarla por la praxis. Bogotá: Tercer Mundo.

» Fogelman, C. y Bassett, T.J. (2017). Mapping for investability: Remaking land and maps in Lesotho. Geoforum, 82, 252-258.

»Freire, P. (2008 [1970]). Pedagogía del oprimido. Buenos Aires: Siglo XXI.

» GEPCyD - Grupo de Ecología Política, Comunidades y Derechos. (2011). Construyendo territorios campesinos. La cartografía social en el conflicto por la tierra. IX Jornadas de Sociología - Pre ALAS. Facultad de Ciencias Sociales, Universidad de Buenos Aires.

»Habegger, S. y Mancila, I. (2006). El poder de la Cartografía Social en las prácticas contrahegemónicas o La Cartografía Social como estrategia para diagnosticar nuestro territorio. Revista Araciega, 14, 1-10.

》Haesbaert, R. (2014). Lógica zonal y ordenamiento territorial: para rediscutir la proximidad y la contigüidad espaciales. Cultura y representaciones sociales, 8(16), 9-29.

" Harley, J.B. (2005). La nueva naturaleza de los mapas: Ensayos sobre la historia de la cartografía. México: Fondo de Cultura Económica.

» Kitchin, R. y Dodge, M. (2007). Rethinking maps. Progress in Human Geography, $31(3), 331-344$. 
» Kitchin, R., Gleeson, J. y Dodge, M. (2012). Unfolding mapping practices: a new epistemology for cartography. Transactions of the Institute of British Geographers, 38(3), 480-496.

» Lefebvre, H. (2009 [1970]). Space and the State. En H. Lefebvre, Space, State, World. Selected Essays (223-253). Minneapolis: University of Minnesota Press.

» Lefebvre, H. (2013 [1974]). La producción del espacio. Madrid: Capitán Swing.

»Lois, C. (2014). Mapas para la nación: episodios en la historia de la cartografía argentina. Buenos Aires: Biblos.

» Lois, C. (2015). Los mapas y las geometrías del espacio. La imagen cartográfica como praxis de la espacialización del pensamiento. Terra Brasilis (Nova Série), Revista da Rede Brasileira de História da Geografia e Geografia Histórica, 8, 1-24.

"Lopes de Souza, M. (2013). Os conceitos fundamentais da pesquisa sócioespacial. Río de Janeiro, Brasil: Bertrand.

» Parque Nacional Iguazú (2017). Plan de Gestión 2017-2023.

» Pickles, J. (2004). A history of spaces: cartographic reason, mapping and the geocoded world. Londres: Routledge.

»Salamanca, C. (2012). Memoria, acción colectiva y narrativas territoriales: paradojas y desafíos de la cartografía social en Argentina. En C. Salamanca y R. Espina (Comps.), Mapas y derechos. Experiencias y aprendizajes en América Latina (pp. 141-194). Rosario: Editorial de la Universidad Nacional de Rosario.

» Scalerandi, V. (2011). La Fábrica en Cabure-í. Trabajadores, campesinos y foresto industria en el Noreste de Misiones 1930-1970. Tesis de maestría en Antropología Social, Universidad Nacional de Misiones, Posadas.

» Soja, E. (2016). La ciudad y la justicia espacial. En B. Bret, P. Gervais-Lambony, C. Hancock y Landy, F. (Comp.), Justicia e injusticias espaciales (pp. 99-106). Rosario: UNR Editora.

»Wood, D. y Fels, J. (2008). The nature of maps. Chicago: Chicago University Press.

\section{Francisco Fernández Romero / franfernandez91@gmail.com}

Licenciado en Geografía (Universidad de Buenos Aires) y doctorando en Geografía (Universidad de Buenos Aires) con beca doctoral CONICET. Su investigación doctoral explora las demandas por el acceso al espacio público por parte del movimiento de mujeres trans y travestis y por parte del movimiento de personas con discapacidad, en Buenos Aires, durante las últimas décadas.

\section{Lucila Muñecas / lucila.munecas@gmail.com}

Licenciada en Geografía (Universidad de Buenos Aires) y doctoranda en Geografía (Universidad de Buenos Aires) con beca doctoral CONICET. Su línea de investigación se centra en políticas ambientales en el norte de Misiones, teniendo en cuenta la incidencia de los discursos y representaciones en torno al ambiente y la conservación y en las tensiones que dichas políticas generan con otras actividades, como la agricultura familiar. 
Francisco Fernández Romero, LUCILA MuñeCAS, Aymara Suyai Zanotti, LUIS Piccinali

\section{Aymara Suyai Zanotti / aymara.zanotti@gmail.com}

Licenciada en Geografía (Universidad de Buenos Aires) y doctoranda en Geografía (Universidad de Buenos Aires) con beca doctoral CONICET. Su investigación doctoral gira en torno al análisis de procesos de producción de hábitat y el ordenamiento territorial en el espacio rural de la provincia de Misiones.

\section{Luis Emilio Piccinali / luispicci@gmail.com}

Licenciado en Geografía (Universidad de Buenos Aires). Docente auxiliar de Sistemas de Información Geográfica del Departamento de Geografía de la Facultad de Filosofía y Letras de la UBA y colaborador de proyectos de investigación del Instituto de Geografía "Romualdo Ardissone" de la UBA. Se desempeña en áreas de desarrollo geomático vinculadas con la gestión pública y desempeñó capacitaciones en la implementación de nuevas infraestructuras de datos espaciales en IDERA. 